\title{
Atendimento às pessoas LGBTQIA+ e a não aplicabilidade dos princípios do SUS: Universalidade, Integralidade e Equidade
}

Care to LGBTQIA+ people and the non applicability of the principles of the SUS: Universality, Integrality and Equity

Servicio a las personas LGBTQIA+ y la no aplicabilidad de los principios del SUS: Universalidad, Integralidad y Equidad

\section{Resumo}

Objetivo: Analisar a produção científica de enfermagem sobre o atendimento à população LGBTQIA+ no serviço de saúde. Materiais e métodos: Trata-se de um artigo de revisão integrativa de literatura sobre a produção científica do atendimento à população LGBTQIA+ no serviço de saúde. Que abrange pesquisas: literatura teórica e metodológica, que apontam os estudos realizados anteriormente possibilitando obter conclusões ao assunto de interesse. Foram incluídos artigos entre 2018 e 2021, indexados nas bases de dados da Biblioteca Nacional de Saúde (BVS), independente do periódico de publicação. Resultados: O Ministério da Saúde vem se preocupando e buscando inserir um acesso humanizado e qualificado para a população LGBTQIA+. A evolução trouxe resultados positivos e uma certa visibilidade, então observou-se que mesmo surgindo várias questões sobre a necessidade de um olhar mais especializado não houve um avanço maior do que a Política Nacional LGBT que foi escrita em 2011. Considerações finais: Apontase como prioridade, por meio desta revisão e todos os aspectos analisados no tocante ao atendimento à população LGBTQIA+ e a não aplicabilidade dos princípios do SUS, a necessidade de mais estudos que envolvam a temática, pode-se reconhecer os riscos que a população LGBTQIA+ está vulnerável, e o quanto se deve melhorar os vínculos para o atendimento, para aperfeiçoar a consulta e alcançar o objetivo que é orientar e se aproximar.

Palavras-chave: Enfermagem; Vulnerabilidade em saúde; Cuidado de enfermagem; Atenção integral à saúde; Pessoas LGBTQIA+.

\section{Abstract}

Objective: To analyze the scientific production of nursing on care for the LGBTQIA+ population in the health service. Materials and methods: This is an integrative literature review article on the scientific production of care for the LGBTQIA+ population in the health service. Covering research: theoretical and methodological literature, which point to the studies carried out previously, making it possible to draw conclusions on the subject of interest. Articles between 2018 and 2021 were included, indexed in the databases of the National Health Library (BVS), regardless of the journal of publication. Results: The Ministry of Health has been concerned and seeking to introduce humanized and qualified 
access for the LGBTQIA+ population. The evolution has brought positive results and a certain visibility, so it was observed that even with several questions arising about the need for a more specialized look, there was no greater progress than the National LGBT Policy that was written in 2011. Final considerations: It points out as a priority, through this review and all the aspects analyzed regarding the service to the LGBTQIA+ population and the nonapplicability of the SUS principles, the need for more studies involving the theme, it is possible to recognize the risks that the LGBTQIA+ population is vulnerable, and how much should be improved the links to the service, to improve the consultation and reach the objective which is to guide and get closer.

Keywords: Nursing; Health vulnerability; Nursing care; Comprehensive health care; LGBTQIA+ People.

\section{Resumen}

Objetivo: Analizar la producción científica de la enfermería sobre el cuidado de la población LGBTQIA + en el servicio de salud. Materiales y métodos: Se trata de un artículo de revisión integradora de la literatura sobre la producción científica de la atención a la población LGBTQIA + en el servicio de salud. Investigación de cobertura: literatura teórica y metodológica, que apunta a los estudios realizados con anterioridad, permitiendo sacar conclusiones sobre el tema de interés. Se incluyeron artículos entre 2018 y 2021, indexados en las bases de datos de la Biblioteca Nacional de Salud (BVS), independientemente de la revista de publicación. Resultados: El Ministerio de Salud se ha preocupado y buscando introducir un acceso humanizado y calificado para la población LGBTQIA +. La evolución ha traído resultados positivos y cierta visibilidad, por lo que se observó que aun con varias preguntas surgidas sobre la necesidad de una mirada más especializada, no hubo mayor avance que la Política Nacional LGBT que se redactó en 2011. Consideraciones finales: Se señala como prioridad, a través de esta revisión y de todos los aspectos analizados en cuanto al servicio a la población LGBTQIA + y la no aplicabilidad de los principios del SUS, la necesidad de más estudios que involucren la temática, es posible reconocer los riesgos que la LGBTQIA + la población es vulnerable, y cuánto deben mejorarse los vínculos con el servicio, para mejorar la consulta y alcanzar el objetivo que es orientar y acercar.

Palabras clave: Enfermería; Vulnerabilidad sanitaria; Cuidado de enfermería; Atención Integral a la salud; Personas LGBTQIA +.

\section{Introdução}

A saúde é um direito universal e fruto de um processo histórico e social homologado na Constituição Federativa Brasileira de 1988, "garantido mediante políticas sociais e econômicas que visem à redução do risco de doença e de outros agravos e ao acesso universal e igualitário às ações e serviços para sua promoção, proteção e recuperação.” (Brasil 1990).

Ainda que preconizado por leis e portarias, com implantação de políticas públicas, onde afirmam que a saúde é um direito de todes ${ }^{1}$, bem como, ter um atendimento humanizado, o acesso a saúde da população Lésbicas, Gays, Bissexuais, Travestis e Transexuais, Queer, Intersexo, Assexuais/Agênero e mais (LGBTQIA+) carrega consigo um histórico de discriminação, marginalização e exclusão.

Em 2011 o Ministério da Saúde (MS) implantou a Política Nacional de Saúde Integral Lésbicas, Gays, Bissexuais, Travestis e Transexuais (LGBT), onde trouxe resultados positivos, visibilidade e inclusão para essa população. Um grande avanço, buscando promoção da saúde, com o objetivo da melhoria do atendimento, sem desigualdade, mas sim, com integralidade e equidade (Brasil, 2011).

A integralidade se apresenta como um conceito de ruptura histórica e institucional que parte de uma crítica a oposição entre as intervenções curativas e preventivas. A Carta Magna do Brasil (Brasil, 1988) declara que a necessidade do atendimento integral deve priorizar as ações de prevenção, não causando prejuízos as ações de assistência. Assim, isso nos implica a afirmar que es usuáries do Sistema Único de Saúde (SUS) têm por direito a atenção das suas necessidades, o que nos direciona ao princípio da equidade, que em seu cerne, é fruto dos maiores problemas sociais brasileiro: as iniquidades sociais e econômicas. Essas iniquidades levam a desigualdades no acesso, na gestão e na produção de serviços de saúde, ao atentar para as necessidades coletivas e individuais, procurando investir onde a iniquidade é maior.

\footnotetext{
${ }^{1}$ Para construção deste projeto, optou-se por utilizar uma linguagem neutra e que não imponha gênero as palavras, portanto, a vogal "e" será utilizada em alguns momentos para possibilitar essa neutralidade. Quando a vogal "e" fizer distinção de gênero, esta será substituída pela vogal "o".
} 
Um estudo de Cabral et al. (2019) traz que a educação em saúde realizada por profissionais não atinge as necessidades encontradas por essa população, pois por muitas vezes, não se considera a orientação sexual e identidade de gênero das pessoas, tendendo aos profissionais não associar as práticas sexuais e seus riscos à saúde, sendo o desconhecimento um dos motivos para deficiência assistencial. Pode-se observar também que o preconceito é contribuinte para o distanciamento entre o profissional e es usuáries. Se faz necessário criar vínculos, assim Crispim et al. (2018) afirma que a enfermagem é a categoria com maior envolvimento nas ações destinadas a assistência, mascarar isso, é negligenciar o atendimento e os riscos a que essa população está vulnerável.

Com isso, o princípio da universalidade e outros princípios são amalgamados pelo conceito de saúde tradicionalmente denominado ampliado. A melhor versão está descrita na definição de saúde da VIII Conferência Nacional de Saúde (VIII CNS), que traz saúde por meio de uma série de condicionantes, como alimentação, moradia, educação, renda, meio ambiente, acesso e posse de terra, e acesso aos serviços de saúde (Ministério Da Saúde, 1986).

Assim, "a universalidade do SUS apresenta-se não apenas como o direito à saúde garantido mediante políticas públicas, bem como aponta para a questão do direito à vida" (Matta, 2007, p. 68) e à "igualdade a assistência da saúde, sem preconceitos ou privilégios de qualquer espécie" (Brasil, 1988). Pode-se observar, que o princípio não é aplicado em sua totalidade, tornandose excludente para uma parcela da população, no caso a população LGBTQIA+, que ao acessar o atendimento, se depara com a discriminação, com isso a não efetivação de um vínculo e um atendimento de qualidade, bem como a possibilidade de não retornar, as tornando mais expostas, vulnerabilizadas.

O conceito de vulnerabilidade propõe o oferecimento de narrativas adicionais ao que se caracteriza como processo saúde-doença-cuidado, pois se necessita fornecer recursos que avaliem as diferentes oportunidades que os indivíduos têm de adoecer e morrer. Gondim (2007) expõe que esses elementos são formados por características singulares do seu cotidiano, prática das dimensões comportamentais, sociais, e político institucionais, sendo elas relevantes ou não para sua exposição a um determinado fator de risco, relacionadas às diferentes suscetibilidades de indivíduos e grupos populacionais.

Evidencia-se nas matrizes curriculares de cursos de graduação em Saúde, a necessidade de gerar produções e discussões que compreendam as especificidades da população LGBTQIA+, visto que a abordagem realizada ainda é incipiente, assim surgindo o interesse pela temática, por observar que ainda é utilizada uma visão reducionista as genitálias, tendo como base o padrão cisheteronormativo, focada em patologias e dificultando uma assistência qualificada a essas pessoas.

Partindo desta assertiva, o presente artigo objetivou identificar a produção cientifica sobre o atendimento à população LGBTQIA+ no serviço de saúde entre os anos de 2018 e 2021 na literatura científica nacional.

\section{Metodologia}

Trata-se de um artigo de revisão integrativa de literatura sobre a produção científica do atendimento à população LGBTQIA+ no serviço de saúde. Que abrange pesquisas realizadas anteriormente, que possibilitam obter conclusões ao assunto de interesse. Foram incluídos artigos entre 2018 e 2021, indexados na base de dados da Biblioteca Nacional de Saúde (BVS), independente do periódico de publicação.

Para a estratégia de busca inicialmente foi verificado se as palavras-chaves escolhidas estavam cadastradas nos Descritores em Ciências da Saúde (DECs), a saber: LGBT; enfermagem; vulnerabilidade em saúde, cuidado de enfermagem e atenção integral à saúde. Após, foram utilizadas combinações dos descritores citados intermediados pelo operador booleano AND, sendo eles: "enfermagem AND LGBT", "vulnerabilidade em saúde AND LGBT", "cuidado de enfermagem AND LGBT", "atenção integral à saúde AND LGBT".

Foram adotados os critérios de inclusão: resumo disponível nas bases de dados, artigos publicados na integra e em língua portuguesa, disponíveis online e gratuitos, ter sido publicado entre 2018 e 2021, que sua abordagem tenha a temática 
proposta. Utilizaram-se as seguintes bases de dados: Literatura Latino Americana e do Caribe em Ciências e Saúde (LILACS), Base de Dados de Enfermagem (BDENF), Sistema Online de Busca e Análise de Literatura Médica (MEDLINE), Scientific Electronic Library Online (SCIELO). Sendo os critérios de exclusão: não estar em formato de artigo, estar fora do período de publicação, fora da temática e em idiomas não nativos.

Como protocolo de tratamento dos dados optou-se pela análise de conteúdo temática, empregado por Minayo (2012) definidas da seguinte maneira: fase exploratória da pesquisa, que se inicia ainda no projeto de investigação com a escolha do material a formulação dos objetivos da análise. O segundo momento que ocorrera a dedicação a coleta de dados onde se reuni as informações que irão responder à pergunta da investigação. E por último o tratamento do material encontrado que se subdivide em três fases.

A primeira delas é a ordenação, onde será realizado o mapeamento do material coletado e sua organização; em seguida será processada à classificação dos dados a partir dos questionamentos que direcionam a pesquisa, identificando o que surge de relevante para a investigação; pôr fim a análise propriamente dita, onde será executada a articulação dos dados e o que já se tem de produção cientifica relacionada ao conteúdo, respondendo as inquietações da pesquisa que serão traçadas a partir do objetivo do estudo (Minayo, 2012).

\section{Resultados}

Ao analisar, separadamente, cada um dos descritores, encontrou-se um elevado número de publicações (Figura 1), onde posteriormente, realizou-se a associação dos descritores citados, a fim de realizar uma aproximação das produções encontradas, ou seja, daquelas que contribuíssem para a elucidação do objetivo apresentado.

A fase seguinte, formada pela leitura minuciosa e exploratória dos artigos selecionados (Quadro 1), permitiu a organização para o último passo, o qual foi localizar o material para compor a bibliografia potencial. Nesta etapa, os trabalhos que não colaboravam com informações adequadas para esse estudo, tais como artigos repetidos e os que não abordassem a temática proposta, foram excluídos.

Figura 1 - Fluxograma do percurso metodológico para a obtenção dos artigos elencados para o estudo.

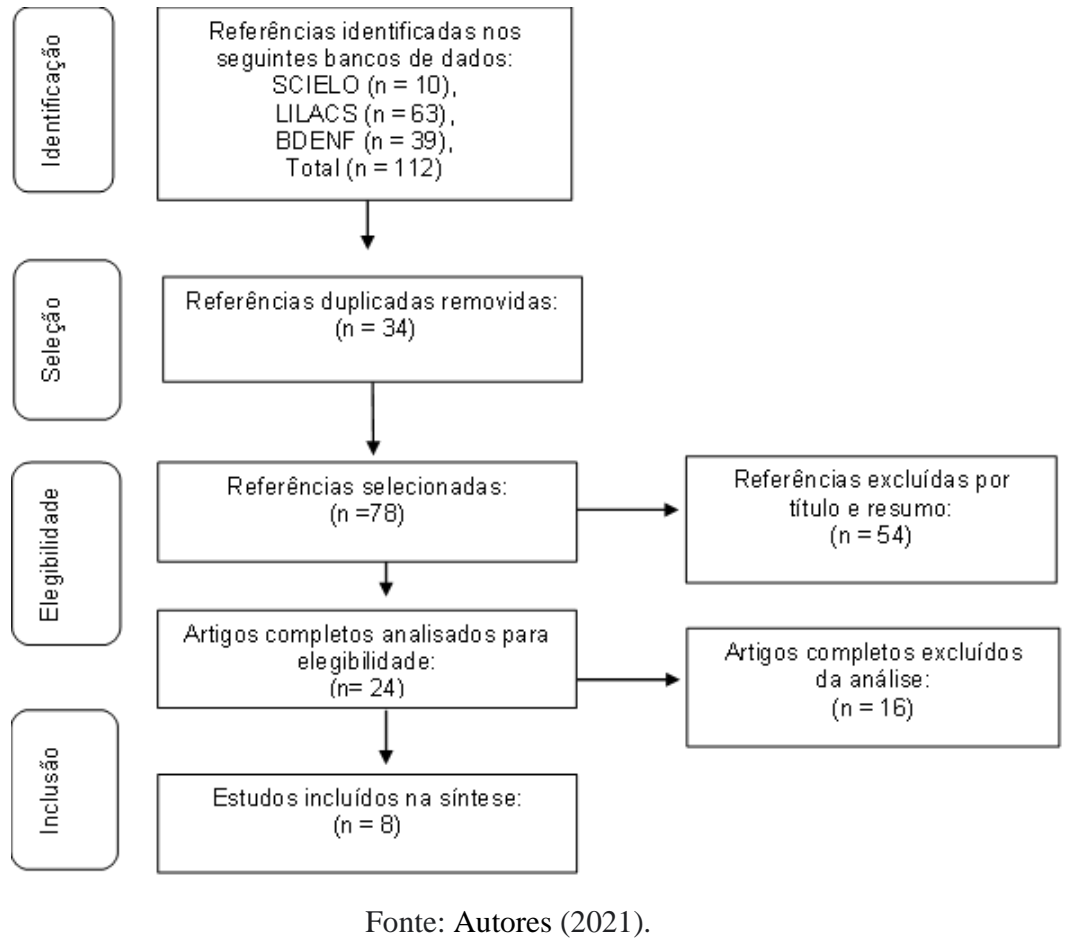


Quadro 1 - Corpus documental de revisão integrativa, 2021.

\begin{tabular}{|c|c|c|c|c|c|}
\hline ID & AUTOR/ANO & TÍTULO & $\begin{array}{l}\text { PERIÓDICO DE } \\
\text { PUBLICAÇÃO }\end{array}$ & MÉTODO & OBJETIVOS \\
\hline 01 & $\begin{array}{l}\text { SILVA et al., } \\
2021\end{array}$ & 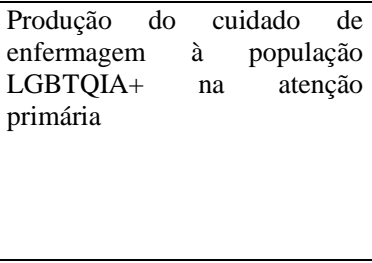 & $\begin{array}{l}\text { Revista de } \\
\text { divulgação científica } \\
\text { sena aires }\end{array}$ & $\begin{array}{c}\text { Estudo } \\
\text { qualitativo }\end{array}$ & $\begin{array}{l}\text { Descrever a produção do cuidado } \\
\text { em Enfermagem à saúde de } \\
\text { Lésbicas, Gays, Bissexuais, } \\
\text { Travestis e Pessoas Trans Queers } \\
\text { Intersexos, Assexuais e outras } \\
\text { identidades sexuais e de gênero, a } \\
\text { partir das reflexões acerca do } \\
\text { trabalho da enfermeira. }\end{array}$ \\
\hline 02 & $\begin{array}{l}\text { SANTANA et } \\
\text { al., } 2020\end{array}$ & $\begin{array}{l}\text { Dificuldades no acesso aos } \\
\text { serviços de saúde por Lésbicas, } \\
\text { Gays, Bissexuais } \\
\text { Transgêneros }\end{array}$ & $\begin{array}{lr}\text { Revista } & \text { de } \\
\text { Enfermagem } & \text { UFPE } \\
\text { On Line } & \end{array}$ & $\begin{array}{l}\text { Estudo } \\
\text { bibliográfico }\end{array}$ & $\begin{array}{l}\text { Analisar as dificuldades de acesso } \\
\text { aos serviços de saúde pelas } \\
\text { pessoas LGBT. }\end{array}$ \\
\hline 03 & $\begin{array}{l}\text { FERREIRA et } \\
\text { al., } 2019\end{array}$ & $\begin{array}{l}\text { "Não tem essas pessoas } \\
\text { especiais na minha área": saúde } \\
\text { e invisibilidade das populações } \\
\text { LGBT na perspectiva de agentes } \\
\text { comunitários de saúde }\end{array}$ & $\begin{array}{l}\text { Revista Eletrônica } \\
\text { de Comunicação, } \\
\text { Informação e } \\
\text { Inovação em Saúde }\end{array}$ & $\begin{array}{c}\text { Pesquisa } \\
\text { qualitativa }\end{array}$ & $\begin{array}{l}\text { Analisar os sentidos atribuídos por } \\
\text { agentes comunitários de saúde } \\
\text { acerca do cuidado em saúde para } \\
\text { as populações LGBT. }\end{array}$ \\
\hline 4 & $\begin{array}{l}\text { PAULINO; } \\
\text { RASERA; } \\
\text { TEIXEIRA; } \\
2019\end{array}$ & $\begin{array}{l}\text { Discursos sobre o cuidado em } \\
\text { saúde de Lésbicas, Gays, } \\
\text { Bissexuais, r Travestis, } \\
\text { Transexuais (LGBT) entre } \\
\text { médicas(os) da Estratégia Saúde } \\
\text { da Família }\end{array}$ & Interface (Botucatu) & $\begin{array}{l}\text { Análise de } \\
\text { discurso }\end{array}$ & $\begin{array}{l}\text { Identificar os discursos sobre o } \\
\text { acesso e a qualidade da atenção } \\
\text { integral à saúde da população de } \\
\text { Lésbicas, Gays, Bissexuais, } \\
\text { Travestis e Transexuais (LGBT) } \\
\text { entre médicas(os) da Estratégia } \\
\text { Saúde da Família, refletindo sobre } \\
\text { como esses discursos podem } \\
\text { impactar o cuidado em saúde da } \\
\text { população LGBT. }\end{array}$ \\
\hline 05 & $\begin{array}{l}\text { CABRAL et al., } \\
2019\end{array}$ & $\begin{array}{l}\text { Assistência de enfermagem às } \\
\text { mulheres Lésbicas e Bissexuais }\end{array}$ & $\begin{array}{lr}\text { Revista } & \text { de } \\
\text { Enfermagem } & \text { UFPE } \\
\text { On Line } & \end{array}$ & $\begin{array}{c}\text { Estudo } \\
\text { qualitativo }\end{array}$ & $\begin{array}{l}\text { Analisar, sob a ótica de mulheres } \\
\text { lésbicas e bissexuais, a assistência } \\
\text { de Enfermagem em Unidades de } \\
\text { Saúde da Família. }\end{array}$ \\
\hline 06 & $\begin{array}{l}\text { BELÉM et al., } \\
2018\end{array}$ & $\begin{array}{l}\text { Atenção à saúde de lésbicas, } \\
\text { gays, bissexuais, travestis e } \\
\text { transexuais na Estratégia Saúde } \\
\text { da Família }\end{array}$ & $\begin{array}{l}\text { Revista Baiana de } \\
\text { Enfermagem }\end{array}$ & $\begin{array}{c}\text { Estudo } \\
\text { qualitativo }\end{array}$ & $\begin{array}{l}\text { Analisar a atenção à saúde } \\
\text { prestada à população de Lésbicas, } \\
\text { Gays, Bissexuais, Travestis e } \\
\text { Transexuais na Estratégia Saúde } \\
\text { da Família. }\end{array}$ \\
\hline 07 & $\begin{array}{l}\text { FARIAS et al., } \\
2018\end{array}$ & $\begin{array}{l}\text { Os cuidados do enfermeiro às } \\
\text { lésbicas }\end{array}$ & $\begin{array}{lr}\text { Revista } & \text { de } \\
\text { Enfermagem } & \text { UFPE } \\
\text { On Line } & \\
\end{array}$ & $\begin{array}{l}\text { Estudo tipo } \\
\text { revisão } \\
\text { integrativa }\end{array}$ & $\begin{array}{l}\text { Identificar como está sendo } \\
\text { abordado o cuidado do enfermeiro } \\
\text { às lésbicas. }\end{array}$ \\
\hline 08 & $\begin{array}{l}\text { OLIVEIRA et } \\
\text { al., } 2018\end{array}$ & $\begin{array}{l}\text { Serviços de saúde para } \\
\text { Lésbicas, Gays, Bissexuais e } \\
\text { Travestis/Transexuais }\end{array}$ & $\begin{array}{lr}\text { Revista } & \text { de } \\
\text { Enfermagem } & \text { UFPE } \\
\text { On Line } & \end{array}$ & $\begin{array}{l}\text { Estudo quanti- } \\
\text { qualitativo }\end{array}$ & $\begin{array}{l}\text { Analisar, sob a ótica de } \\
\text { profissionais da Equipe Saúde da } \\
\text { Família, o acesso de Lésbicas, } \\
\text { Gays, } \quad \text { Bissexuais à } \\
\text { Travestis/Transexuais } \\
\text { Unidades Básicas de Saúde da } \\
\text { Família. }\end{array}$ \\
\hline
\end{tabular}

Fonte: Autores (2021).

\section{Discussão}

O Ministério da Saúde (MS) vem se preocupando e buscando inserir um acesso humanizado e qualificado para a população LGBTQIA+. A evolução trouxe resultados positivos e uma certa visibilidade, então observou-se que mesmo surgindo várias questões sobre a necessidade de um olhar mais especializado não houve um avanço maior do que a Política Nacional LGBT que foi escrita em 2011, atualmente as pessoas ainda vivenciam preconceito e discriminação ao procurar o atendimento.

O estudo de Morais Filho et al. (2019) acredita que partindo de conceitos de uma saúde ampliada, rompendo a educação biologista, com reducionismo à genitalidade e um pensamento distante onde o conceito saúde seria completo bem-estar, conteúdo tão comum nas matrizes curriculares, de certa forma acabam se tornando padrão na assistência, levando parâmetros para as ações do cotidiano, deixando assim de executar a singularidade da promoção e prevenção em saúde para a população LGBTQIA+.

Para uma modificação deste parâmetro Nietsche et al. (2018) propõe que a implantação do tema na matriz curricular da enfermagem permite ao profissional uma visão ampla do atendimento das diversidades presente no contexto social atual, fazendo com que se desenvolvam estratégias e questionem a ideia de heteronormatividade do sistema. Desse modo, profissionais iriam 
atuar com menos dilemas socioculturais, julgamentos de valores e juízos, aproximando-os das demandas reais que a prática exige.

Analisando a equidade em saúde e sua necessidade dentro da assistência, se dá ênfase a ideia de não discriminação, galgando a não marginalização por raça/cor/etnia, mas houve a necessidade de incorporar outros aspectos como vulnerabilidade social, religião, gênero e sexualidade, gerando novas políticas que se aproximem do conceito de equidade (Siqueira, Hollanda; Motta, 2017).

Para Silva et al. (2021) a inclusão da população LGBTQIA+ no cuidado à saúde precisa de grandes transformações, seja no modo de pensar dos profissionais, bem como em sua atuação. Como recomendado por leis e portarias, já existe a implantação de políticas públicas, onde se afirma que a saúde é um direito de todes, preconizado pelo atendimento humanizado, acesso a saúde da população com autonomia, sendo respeitados independente de orientação sexual ou identidade de gênero.

Essas transformações necessitam de investimentos em políticas públicas de qualidade e que contemplem as necessidades da população em questão, execução de ações em saúde em conjunto a essas pessoas, o que lhes proporciona a ocupação do seu lugar de direito, assegurados pelos princípios do SUS e busca a implantação de estratégias para a mudança no sistema que ainda é exclusivamente heteronormativo nas suas práticas.

Para a efetivação de estratégias de atenção à saúde da população LGBTQIA+ o estudo de Farias et al. (2018) destaca a importância do vínculo, onde es usuáries se sintam acolhides e que seja realizado um atendimento sem preconceito, através de uma assistência não heteronormativa, praticando então a equidade e universalidade. Preconceito este, apontado como uns dos principais motivos de distanciamento entre profissionais e população, existindo a necessidade de desconstrução desses preconceitos enraizados pela sociedade para que a assistência não seja prejudicada.

Sobre a relação entre tempo de atuação e formação, os achados de Oliveira et al. (2018) revelam que o tempo em que os profissionais estavam exercendo a profissão é maior que dez anos, em sua maioria, o que traz a necessidade de atualização, capacitação e aprimoramento, levando em conta o tempo de experiência. Podendo ser auxiliados em aperfeiçoamento e em atualização de práticas, significados e padrões sociais vigentes, que podem reproduzir retrocessos que podem reforçar a situação de vulnerabilidade dessas pessoas.

Ratificando, Cabral et al. (2019) entende esse reforço de vulnerabilidade como reflexo de uma educação em saúde que não atinge as necessidades dessas pessoas, ou saberes equivocados devido ao próprio retrocesso em seus conhecimentos acerca dessas necessidades.

Para Silva e Prata (2018) as construções sociais de algumas narrativas trazidas até a atual conjuntura são históricas, carregadas de estigmas e discriminação, o que geram barreiras na compreensão de todo o processo de existência dessa população, conceitos vindos a partir de normas e padrões hegemônicos que vê e pensa a sexualidade como exclusivamente heterossexual, contribuindo para seu adoecimento e invisibilidade.

A invisibilidade trazida por Ferreira et al. (2019) retrata bem esse não lugar dessas pessoas, carregado de violências e negligências que interferem diretamente na qualidade de vida e na assistência prestada aos mesmes. Assistência essa, pautada apenas na patologia, onde as pessoas precisam necessariamente estar doentes, fazendo associação muitas vezes a Infecções Sexualmente Transmissíveis (ISTs) e estigmas trazidos historicamente.

Esse não lugar e os estigmas arrastados até a atual conjuntura também são expostos em Belém et al. (2018) que retrata a baixa adesão desses usuáries a Atenção Básica relacionada ao receio de sofrer discriminação não só pelos profissionais, mas também pelas outras pessoas que estão para ser atendidas. O não pertencimento se dá exatamente pela ideia de que esses corpos não sejam agentes de promoção e prevenção, apenas de tratamento e visto que sua orientação referida é uma barreira para comunicação com o profissional. 
Sustentar discursos a patologização de identidades e práticas sexuais fora do padrão em que a sociedade entende como norma, só legitima silogismos e reproduz violências. Paulino, Rasera e Teixeira (2019) confirma isso em seu estudo ao trazer a necessidade da quebra desses estigmas históricos que associam a população LGBTQIA+ como pertencentes de uma categoria que se reforça por meio de alegações científicas.

Destacando a omissão de orientação sexual ou identidade de gênero na busca por esses profissionais Santana et al. (2020) demonstram que o medo da repulsa faz com que evitem o acompanhamento em serviços de saúde. A dificuldade em identificar fatores que prejudicam o processo saúde-doença-cuidado impossibilitam o profissional de um cuidado não biologista e integral, não extraindo assim dados fundamentais para o atendimento singular e de qualidade.

Pois, estas informações cedidas nos prontuários durantes as consultas, geram dados epidemiológicos para o sistema onde possibilitam uma gestão estratégica e melhoria na efetivação dos serviços. Quando existe omissão dos dados por falta de preenchimento, muitas vezes por achar que não é relevante perguntar, as informações ficam imprecisas para uma análise de pesquisa onde podem gerar investimentos e amplitude do campo. Informações essas que não são obrigatórias ainda nas consultas, mas que são de extrema necessidade para uma melhoria na assistência.

A existência dessa população precisa ser respeitada, cada letra do acrônimo representa milhões de pessoas que estão à mercê de um sistema machista, opressor onde a heteronormatividade dita a ideia equivocada de que todas as pessoas são ou devem ser heterossexuais, considerando tudo fora disso um desvio, que não pode ser respeitado.

\section{Considerações Finais}

Aponta-se como prioridade, por meio desta revisão e todos os aspectos analisados no tocante ao atendimento à população LGBTQIA+ e a não aplicabilidade dos princípios do SUS, a necessidade de mais estudos que envolvam a temática, comprovando que profissionais de enfermagem necessitam de aptidão para exercer sua função do cuidado, se capacitando para lidar com a pluralidade dessas pessoas e de suas demandas.

A partir desta revisão pode-se reconhecer os riscos que a população LGBTQIA+ está vulnerável, e o quanto se deve melhorar os vínculos para o atendimento, para aperfeiçoar a consulta e alcançar o objetivo que é orientar e se aproximar. Importante salientar a necessidade de realizar educação em saúde para essas pessoas nos atendimentos, bem como educação permanente e continuada para profissionais lidarem com a população.

Faz-se necessária a implantação na formação profissional de uma abordagem mais extensa, que desconstrua preconceitos enraizados pela sociedade machista e pela heteronormatividade da assistência, incentivando profissionais a compreenderem as questões das especificidades das pessoas que compõe a população estudada.

Ressalta-se que profissionais reconheçam a importância da temática e do cuidado, encorajem mudanças no processo de atendimento estimulando cada vez mais o entendimento para essa população e suas necessidades específicas, desenvolvendo a quebra de padrões e pensamentos plurais, para uma sociedade que não é singular.

Desse modo, muito se precisa avançar na produção científica, assim como na assistência à saúde desses indivíduos, para que deixe de ser excludentes em seus atendimentos e acolhimentos, omissão de dados, constrangimentos por falta de conhecimento a acerca do atendimento e promover um acesso de qualidade aplicando os princípios do SUS nesses serviços.

Diante dos fatos mencionados anteriormente, salienta-se a importância do presente estudo gerar

a realização de mais pesquisas, com o escopo de ampliar ainda mais o conhecimento acerca do Atendimento às Pessoas LGBTQIA+ e a Não Aplicabilidade dos Princípios do SUS: Universalidade, Integralidade e Equidade. 


\section{Referências}

Belém, J. M. (2018). et al. Atenção à saúde de lésbicas, gays, bissexuais, travestis e transexuais na estratégia saúde da família. Revista Baiana de Enfermagem, 32.https://doi.org/10.18471/rbe.v32.26475.

Brasil. (1990). [Constituição (1988)]. Constituição da República Federativa do Brasil: promulgada em 5 de outubro de 1988. (4a ed.), Saraiva.

Brasil. (2011). Portaria $n^{\circ}$ 2.836, de $1^{\circ}$ de dezembro de 2011. Institui, no âmbito do Sistema Único de Saúde (SUS), a Política Nacional de Saúde Integral de Lésbicas, Gays, Bissexuais, Travestis e Transexuais (Política Nacional de Saúde Integral LGBT). Diário Oficial [da] União. https://bvsms.saude.gov.br/bvs/saudelegis/gm/2011/prt2836_01_12_2011.html.

Cabral, K. T. F. (2019). et al. Assistência de enfermagem às mulheres lésbicas e bissexuais. Rev. enferm. UFPE on line, 79-85. https://doi.org/10.5205/1981-8963v13i01a237896p79-85-2018.

Crispim, J. E. B. (2018). et al. Assistência de enfermagem à mulher lésbica e bissexual na atenção básica: protocolo de atendimento. Revista de Pesquisa Cuidado é Fundamental Online, 10, 34-39. https://doi.org/10.9789/2175-5361.2018.v10iEspecial.34-39.

Farias, G. M. (2018). et al. Os cuidados do enfermeiro às lésbicas. Rev. enferm. UFPE on line, 2825-2835. https://doi.org/10.5205/1981-8963v12i10a236414p2825-2835-2018.

Ferreira, B. de O. (2019). et al. "Não tem essas pessoas especiais na minha área": saúde e invisibilidade das populações LGBT na perspectiva de agentes comunitários de saúde. Revista Eletrônica de Comunicação, Informação e Inovação em Saúde, 13 (3). https://doi.org/10.29397/reciis.v13i3.1703.

Gondim, G. M de M. (2007). Do conceito de risco ao da precaução: entre determinismos e incertezas. In: Fonseca, A.F.; Corbo, A. M. D’. (Org.). O território e o processo saúde-doença. Rio de Janeiro: EPSJV/FIOCRUZ. (Coleção Educação Profissional e Docência em saúde: a formação e o trabalho do agente comunitário de saúde, 1), 87-120.

Matta, G. (2007). Princípios e diretrizes do Sistema Único de Saúde. In: Matta, G.; Pontes, A.L de M. (org.). Políticas de saúde: organização e operacionalização do Sistema Único de Saúde. Rio de Janeiro: Escola Politécnica de Saúde Joaquim Venâncio/Fiocruz, 61-80.

Moraes Filho, I. M de. (2019). et al. O papel da enfermagem no rompimento dos preconceitos LGBT nos serviços de saúde. Revista de Divulgação Científica Sena Aires, 8 (3), 242-245. https://doi.org/10.36239/revisa.v8.n3.p242a245.

Minayo, M.C de S. (2012). Análise qualitativa: teoria, passos e fidedignidade. Ciência \& saúde coletiva, 17, 621-626. https://www.scielo.br/j/csc/a/39YW8sMQhNzG5NmpGBtNMFf/?lang=pt\&format=pdf.

Ministério da Saúde. (1986). VIII Conferência Nacional de Saúde - relatório final. Ministério da Saúde.

Nietsche, E. A. (2018). et al. Formação do enfermeiro para o cuidado à população homossexual e bissexual: percepção do discente. Revista Baiana de Enfermagem, 32, e25174. https://doi.org/10.18471/rbe.v32.25174.

Oliveira, G. S. (2018) et al. Serviços de saúde para lésbicas, gays, bissexuais e travestis/transexuais. Rev. enferm. UFPE on line, 2598-2609. https://doi.org/10.5205/1981-8963-v12i10a237014p2598-2609-2018.

Paulino, D. B. \& Rasera, E. F. (2019). et al. Discursos sobre o cuidado em saúde de Lésbicas, Gays, Bissexuais, Travestis, Transexuais (LGBT) entre médicas (os) da Estratégia Saúde da Família. Interface-comunicação, saúde, educação, v. 23. https://doi.org/10.1590/Interface.180279.

Santana, A. D da S. (2020). et al. Dificuldades no acesso aos serviços de saúde por lésbicas, gays, bissexuais e transgêneros. Rev. enferm. UFPE on line, 11-12. https://doi.org/10.5205/1981-8963.2020.243211.

Sousa, F. B. \& Sousa, P. M. L. S. (2021). Saúde LGBTQIA+: a vulnerabilidade das minorias sexuais. Research, Society and Development, $10(13)$, e273101321241. https://doi.org/10.33448/rsd-v10i13.21241

Siqueira, S. A.V de. \& Hollanda, E. (2017). Políticas de Promoção de Equidade em Saúde para grupos vulneráveis: o papel do Ministério da Saúde. Ciênc. saúde coletiva, Rio de Janeiro, v. 22 (5), 1397.

http://www.scielo.br/scielo.php?script=sci_arttext\&pid=S141381232017002501397\&lng=pt\&nrm=iso.

Silva, A das N. \& Prata, M. (2018). O atendimento à mulher lésbica e a influência da heteronormatividade no cuidado. Monografia (Aperfeiçoamento/Especialização em Especialização em Direitos Humanos, Gênero e Sexualidade) - Escola Nacional de Saúde Pública Sérgio Arouca.

Silva, A. A da C. (2021). et al. Produção do cuidado de enfermagem à população LGBTQIA+ na atenção primária. Revista de Divulgação Científica Sena Aires, 10 (2). 291-303. https://doi.org/10.36239/revisa.v10.n2.p291a303. 